\section{Acompañando en el último viaje: vivencia de personas mayores institucionalizadas}

\section{Accompanying in the last trip: experience of institutionalized elderly people}

Camila Villarroel Fuenzalida ${ }^{1}$

Miriam Rubio Acuña 2,*

Francisca Márquez Doren ${ }^{3}$

1. Enfermera. Candidata a Magíster en Enfermería. Santiago. Chile.

2. Enfermera. Magíster en Enfermería. Profesor asistente en la Escuela de Enfermería. Pontificia Universidad Católica de Chile. Santiago. Chile.

3. Enfermera-Matrona. Magíster en Enfermería. Doctora en Enfermería. Profesor asociado. Escuela de Enfermería. Pontificia Universidad Católica de Chile. Santiago. Chile.

*Autor para correspondencia.

Correo electrónico: merubioa@uc.cl (Miriam Rubio Acuña).

Recibido el 23 de julio de 2019; aceptado el 3 de septiembre de 2019.

\section{RESUMEN}

El envejecimiento trae consigo pérdidas en diferentes aspectos de la vida, y el proceso de muerte es uno de los más relevantes debido a su mayor proximidad. Un contexto especial que considerar son los establecimientos de larga estancia (ELEAM), lugar en el que viven de manera recurrente el duelo

de compañeros, temática que se encuentra escasamente estudiada a nivel mundial. Objetivo: Comprender los significados de la experiencia vivida por personas mayores institucionalizadas ante el fallecimiento de un compañero residente. Metodología: Se utilizó un diseño cualitativo fenomenológico.

Resultados: Se entrevistó a 13 personas mayores institucionalizadas. El

fenómeno fue dividido en ocho categorías interrelacionadas: día a día y recambio; sentimientos ante el sufrimiento y fallecimiento de un compańero/a; rituales de despedida; muerte como proceso natural y de autorreflexión; soledad; adaptación a la perdida junto a compañeros; rol de las cuidadoras; profesionales, y escasa percepción de apoyo. Conclusión: Los relatos permiten

comprender el proceso que viven las personas mayores al experimentar el fallecimiento de un compañero/a, cómo expresan sus sentimientos y cómo los despiden. Esto les permite reflexionar sobre su propia muerte y sus redes de apoyo. Este conocimiento puede aportar a la comprensión del fenómeno y a la propuesta de cuidados de enfermería basados en las necesidades de cada residente ante el fallecimiento de compañeros.

PALABRAS CLAVE: Anciano, residencia de ancianos, duelo.

\section{ABSTRACT}

Aging is accompanied with losses in differents aspects of life and one of the greater impact is about death, because of its proximity. A important context to consider is (LSE) Long Stay Establishments, place that patients live with frecuency peers bereavement. Objective: Understand the meanings of the experience lived by institutionalized elderly people due to the death of a partner. Methodology: For this study it used a qualitative phenomenological design. Results: 13 elderly people interviewed who established in a institution. The phenomenon was separated in 8 categories connected: day by day and replacement; feelings about suffering and peer's death; farewell rituals; death as a natural process and self-reflection; loneliness, adaptation to the loss with their peers; caregiver's role; professionals and poor perception of support. Conclusion: Their experiences allow to comprehend the process that elder people lives in nursing home to experiment death's peer, their perceptions about their feelings and how they said goodbye to them. This situation makes that elder people reflect about their own death and support networks. This knowledge can contribute to the understanding of the phenomenon and can be useful as guide to make nursing care based on resident's needs when they have to deal with peers death.

\section{- INTRODUCCIÓN}

El envejecimiento es una etapa de la vida en constante cambio, como son los dominios sociales y existenciales ${ }^{1}$. El proceso de la muerte es uno de los temas que adquiere relevancia debido a que la población anciana experimenta más el fallecimiento de pares y seres queridos que otros grupos etarios ${ }^{2}$. Esta situación puede ser más intensa en contextos vulnerables, como la marginación social y/o familiar, lo que manifiesta un empobrecimiento progresivo de las redes de apoyo que posee la persona mayor ${ }^{1,3}$.

Uno de los contextos vulnerables son los establecimientos de larga estancia de personas mayores (ELEAM), los cuales se encuentran legislados en el decreto 14 establecido en el año $2011^{4}$. En estas instituciones residen personas que, por motivos biológicos, psicológicos o sociales, requieren de un medio ambiente protegido y cuidados diferenciados para el mantenimiento de su salud y funcionalidad, influyendo de ma- nera importante en la expectativa de la vida de las personas mayores ${ }^{5}$. En ellas, las personas mayores viven de manera recurrente la pérdida y duelo de compañeros residentes, por lo que se hace vital su adecuado acompańamiento y cuidado.

Lamentablemente, la forma en que las personas mayores viven la experiencia de duelo ha sido escasamente estudiada. Más aun, la información disponible se encuentra enfocada a la pérdida conyugal en un ambiente familiar y domiciliario, por lo que difiere de la realidad vivida en un establecimiento de larga estancia.

En consecuencia, este estudio tiene por objetivo comprender los significados de la experiencia vivida por personas mayores institucionalizadas ante el fallecimiento de un compañero residente, y así contribuir con conocimiento comprensivo que oriente acciones de cuidado y acompańamiento que incorporen la perspectiva de la persona mayor y sus necesidades. 


\section{- METODOLOGÍA}

Se realizó una investigación cualitativa fenomenológica, la cual proporciona el marco para descubrir y comprender la estructura o esencia del fenómeno vivido ${ }^{6}$.

La selección de los participantes se realizó a través de muestreo intencionado, es decir, se buscó a las personas que portaban el fenómeno en estudio. Los criterios de inclusión utilizados fueron: personas mayores institucionalizadas en un ELEAM, haber experimentado la pérdida de un compańero en un periodo mayor de 6 meses, y obtener una puntuación normal en la Escala Yesavage ${ }^{7}$ y Mini-Mental abreviado ${ }^{7}$. Se excluyeron usuarios con importantes problemas de lenguaje que impedían la transmisión del fenómeno.

Se invitó a participar a las personas mayores explicándoles el propósito de la investigación y se solicitó su participación por medio de un consentimiento informado. Se realizaron entrevistas en profundidad en la propia institución, que fueron grabadas, transcritas textualmente y codificadas para mantener la confidencialidad.

Se realizó un análisis interpretativo junto con la recolección de los relatos. A través de este proceso, se logró desvelar la estructura del fenómeno y desarrollar una descripción formal de este una vez saturadas las unidades de significado ${ }^{6}$, lo cual se alcanzó en la sexta entrevista. Además, se realizó la correspondiente triangulación junto a dos investigadoras ${ }^{6}$.

El rigor metodológico se cauteló utilizando los criterios de Guba: la credibilidad se obtuvo mediante la devolución de los participantes (member check) ${ }^{8}$; para aplicar la fiabilidad, se realizaron transcripciones textuales de cada entrevista y, además, se utilizaron narrativas de los testimonios para ejemplificar las esencias ${ }^{8}$.

En cuanto a la auditabilidad, esta se alcanzó al describir detalladamente la metodología con el objetivo de permitir reproducir los procesos implementados en la investigación'; mientras que para aplicar la transferibilidad se detallaron las características de los participantes con el propósito de permitir transferir los resultados encontrados con una población similar ${ }^{8}$.
Durante este estudio se resguardaron los principios éticos ${ }^{10}$ y se consideraron a través de los 7 requisitos de Emanuel ${ }^{11}$. Esta investigación contó con la aprobación del Comité de Ética de la Facultad de Medicina de la Pontificia Universidad Católica de Chile.

\section{RESULTADOS}

Se entrevistó a 13 participantes, de los cuales cinco eran hombres y ocho, mujeres, cuyas edades oscilaban entre los 66 y los 86 años. Todas las personas mayores entrevistadas vivían hacía por lo menos 2 años en el centro de larga estancia y habían presenciado y/o vivenciado el fallecimiento de al menos un compañero.

Con respecto a la red familiar, 7 mujeres no tuvieron hijos ni esposo, mientras que solo una participante era viuda y mantenía contacto continuo con su hijo y sus nietos. En el caso de los hombres, todos conformaron un grupo familiar. No obstante, solo uno refirió mantener contacto por vía telefónica.

En relación con el fenómeno, este emerge como una vivencia en la que las personas mayores residentes en un ELEAM experimentan de manera recurrente el recambio de compañeros debido al fallecimiento de estos, lo cual genera pesar, tristeza y ańoranza (fig. 1). Refieren que todos merecen ser despedidos, sean buenos o malos, ya que quieren acompañarlos en su último viaje. En este contexto, consideran la muerte como un proceso natural y reflexionan sobre su propio fallecimiento. Además, valoran el rol cercano de las cuidadoras y visualizan distantes al resto de los profesionales. El apoyo de los compańeros toma un rol fundamental; sin embargo, es este el que se reduce con el fallecimiento de sus integrantes.

En concordancia con lo anterior, se desvelaron ocho categorías comprensivas: dia a dia y recambio; sentimientos ante el sufrimiento y fallecimiento de un compañero; rituales de despedida; muerte como proceso natural y de autorreflexión; soledad; adaptación a la pérdida junto a compañeros; rol de las cuidadoras; profesionales, y escasa percepción de apoyo.

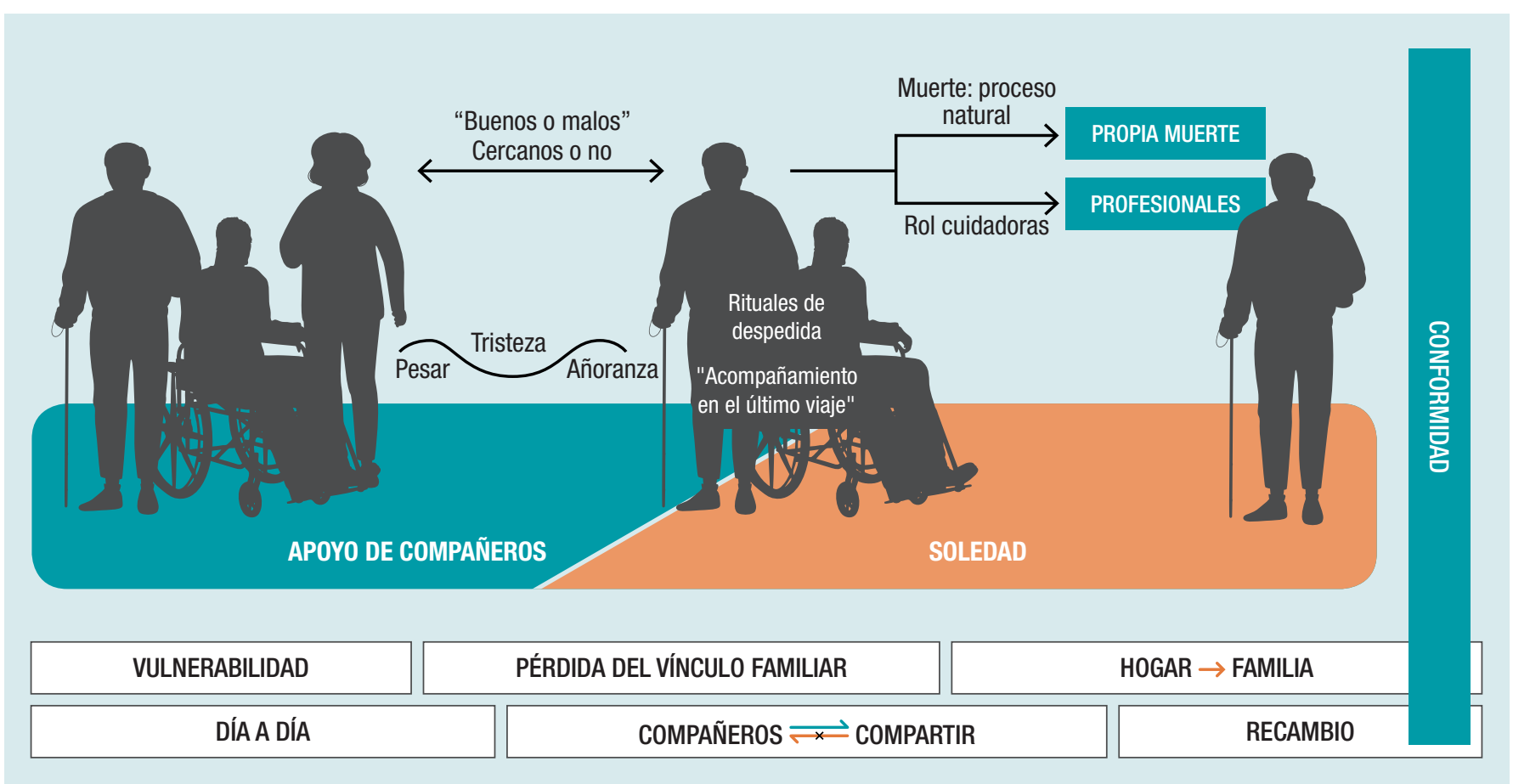

Figura 1. Significado de la experiencia vivida del fallecimiento de un compañero residente. 
En los ELEAM transcurre el día a día de los participantes, donde comparten comidas, celebraciones, actividades, etc. Por otra parte, señalan que el permanente recambio de residentes suele ser brusco y poco amigable para ellos:

“... hay confianza, conversábamos cosas de mujeres, cosas que nos hubiera gustado hacer o de cualquier lesera, pero era diferente, comíamos juntas, compartíamos todos los días..." (E2F2).

"... uno comparte todos los días, en todas las comidas, para las actividades, cuando celebran los cumpleaños” (E11M4).

“... igual cambia todo rápido acá porque les sacan sus cosas rápido y las dejan en la bodega y traen a otra persona, así que nunca se ve sola la pieza. Se muere uno y reciben al tiro a otro..." (E2F2).

“... uno recién está pensando que su amiga se fue y se llevan todo y llega rapidito alguien más, como que me produjo algo cuando pasa, lo encontré triste..." (E10F7).

Una de las principales formas de recambio se debe al fallecimiento de residentes. Al relatar sobre dicha vivencia, surgen los sentimientos ante el sufrimiento y el fallecimiento de un compañero con el cual convivían, siendo la tristeza, el pesar y la añoranza ejes primordiales, pues eran parte de su vida cotidiana y de su nueva familia. Cuando el fallecido es cercano, estos sentimientos se prolongan e intensifican:

“... Da pena cuando son buenas amigas y cuando no, también da, rogamos por ellas. Todos hemos sentido un profundo pesar porque se va alguien, peor si es alguien buena, se sienten más, queda un vacío..." (E1F1).

"... Me la sufrí mucho, porque era mi amiga, le contaba mis cosas y ella me contaba lo suyo. La pena me duro mucho tiempo porque ya no estaba ahí, me costó acostumbrarme..." (E10F7).

A pesar de lo mencionado, consideran la muerte como un descanso para aquellos compañeros que habían padecido dolores y constante deterioro, aliviando el propio sentir:

“... sí, sufrió mucho, daba mucha pena verla. Uno sabe que estaba sufriendo mucho, muchos dolores, uno como que siente un alivio por así decir, pero también mucho pesar..." (E1F1).

“...rezaba por ella para que se sintiera mejor o para que ya pudiera descansar, porque estaba sufriendo mucho. A esas alturas todos queríamos que descansara no más, era terrible verla..." (E2F2).

Ante estas pérdidas, los rituales de despedida adquieren un rol fundamental para las personas mayores, ya que consideran que toda persona lo merece. Estos ritos empiezan con la preparación física del fallecido, realizan un responso, y finalizan con un cortejo fúnebre y su respectivo entierro. Este proceso genera consuelo en quienes lo presencian y, en consecuencia, les permite acompańarlos en su último viaje:

“... le ponen su mejor ropita, los peinan y las maquillan para que se vean mejor. Se lo llevan a la capilla. Después al cementerio, hay unos buses chicos y los que quieren ir, van..." (E6M2).

“... varios fueron a despedirla a su velorio, se da la oportunidad de acompañar en el último viaje. Es bonito que se pueda hacer eso. Acá estamos en la capilla, se hace todo lo que tiene que hacerse, los rezos es una forma importante para mí de despedirlos..." (E0F0).

El vivenciar la pérdida de un compañero residente los hace pensar sobre la muerte como un proceso natural, pues conviven con personas de su misma edad:
"... sabemos que de repente una que otra se tiene que ir, es algo que ocurre continuamente, todos venimos a eso, es parte del hogar y es la ley de la vida..." (E1F1).

"¿vio en la entrada?, somos puros viejos, así que no se puede esperar otra cosa, todos estamos esperando para ver cuando nos toca..." (E10F7).

Sumado a lo anterior, el relatar su experiencia les permite reflexionar sobre su propia muerte y en cómo desean que esta ocurra. Anhelan morir en paz, sin sufrimiento y de forma rápida. Más aun, necesitan saber que partirán acompañados y que serán debidamente despedidos:

"Me puse a pensar cuánto me quedaría. Si es que sería rápido o sería algo sufrido, cosa que no me gustaría nada. Ojalá sea de la mejor forma dentro de lo que se puede..." (E6M2).

"... no me gustaría estar solita, me gustaría que me acompañaran porque ya sé que mi familia no va a estar..." (E10F7).

Al visualizar el contexto en el que se encuentran, la soledad se manifiesta en sus relatos, ya que se pierden integrantes que conforman su red de apoyo y, además, reviven la pérdida del vínculo familiar de quienes viven allí:

“... se van sin familia. Algunos tienen hasta hijos, pero igual la soledad llega. Por eso, prefiero ir a acompañar. Me da pena cuando quedan solos..." (E10F7).

"... a uno ya le da pena que se vayan porque los va a extrańar, pero también como que duele, como que me aprieta algo pensando que se van solos, sin la familia..." (E11M4).

A pesar de ello, las personas mayores perciben el apoyo mutuo entre residentes, dando paso a la adaptación a la pérdida junto a compañeros. Se consuelan entre ellos y participan en la despedida del fallecido. No obstante, es este mismo círculo el que se va reduciendo:

"... nos apoyamos las unas a las otras, conversamos, las recordamos, rezamos. Yo creo que está bien lo que hacemos, nos apoyamos...” (E1F1).

“... era parte del grupo, así que nos dio mucha pena; la recordamos siempre, incluso al principio me daba pena ir al club porque ya no estaba. Así que cuando pasó, nos corrían sus lagrimones y nos acordábamos de cómo era ella..." (E8F6).

A medida que relatan su experiencia, los residentes perciben el rol de las cuidadoras más cercano en comparación con otros trabajadores del lugar, puesto que son quienes ayudan en la preparación del fallecido y de quienes participan. Destacan la importancia de convivir a diario con ellas y los cuidados que ellas les brindan y cómo también se ven afectadas por el fallecimiento de un residente:

“... a algunas cuidadoras les da pena. Ellas los visten, los arreglan para que se vean, eso se agradece. Nos ayudan a nosotros igual, nos llevan a la misa, nos acompañan..." (E9M3)-

“... acá también se preocupan de uno, la ayudan, la atienden. Claro que sí, no me sentí tan sola, me daban un abrazo y algún carińo. Es bonito cuando se preocupan por uno..." (E10F7).

Por el contrario, perciben de forma distante a los profesionales, debido a su escasa participación de los responsos. A pesar de expresar su sentir, las personas mayores no lo consideran cercano. No obstante, justifican 
que están ocupados y que debido a ello no pueden participar en los rituales de despedida.

“... no nos acompañan mucho, se tienen que quedar a cuidar. Pero eso es así no más, no creo que vaya a cambiar. Cuando velan en el salón, pasan, pero no se quedan..." (E2F2).

“... algunas si nos acompañan. Nos acompañan un ratito o nos ayudan a ir no más. No son mala gente, pero están en sus cosas y van un ratito y siguen con sus cosas..." (E10F7).

Al finalizar su relato, realizan un análisis de su vivencia ante la cual refieren desesperanza; sin embargo, se expresan conformes con el accionar del hogar:

“...creo que está todo bien, no se puede hacer más tampoco, fuera de lo que hacen, está dentro de sus posibilidades. El hogar ayuda con los recursos que tiene..." (E3M1).

"...este no es un lugar de muchos recursos y yo creo que no hay mucho más que hacer, así que creo que está bien...” (E8F6).

\section{- DISCUSIÓN Y CONCLUSIÓN}

Para una persona mayor, el fallecimiento de un compañero o pareja constituye la pérdida de alguien con quien compartía las mismas situaciones o experiencias ${ }^{12}$; por tanto, representa la pérdida de una parte de sí mismo ${ }^{13}$, lo cual se ve aumentado en el contexto de vulnerabilidad en el que se encuentran las personas mayores institucionalizadas, las que comparten a diario y poseen similares historias de vida.

Los hallazgos representan la importancia del ambiente en el que se encuentra insertos, pues conforman la vida cotidiana que les permite compartir día a día, la sensación de soledad que se manifiesta tanto al ingresar en el establecimiento como cuando fallece la persona mayor, y a su vez, el rol de cuidadoras y profesionales que influye en la percepción de apoyo de los residentes. Esto no ha sido descrito en estudios similares, por lo que constituye un aporte para el manejo del duelo en personas mayores institucionalizadas.

Dentro de los relatos, los residentes destacan los sentimientos ante la pérdida como tristeza, pena y pesar, los cuales son compartidos entre compańeros. Esto concuerda con la evidencia, puesto que se señala que el dolor es parte del proceso, ya que se considera como un importante impacto, debido a que se asemeja a la pérdida de una parte del alma y el dolor impide ver de manera objetiva la situación ${ }^{14}$. Además, los pensamientos intrusivos, la tristeza y el anhelo son prácticamente sentimientos universales en las primeras etapas del duelo ${ }^{15}$.

Adicionalmente, en los diversos relatos se estableció que el pesar aumentaba cuando fallecía un residente cercano, lo cual se evidencia en diversos estudios, debido a que la muerte de un ser querido se considera una experiencia emocional muy diferente a la habitual, afectando al proceso de duelo ${ }^{14,16}$. Diversos especialistas consideran que uno de los duelos relativamente menos dolorosos es el que se vive cuando el ser querido enferma y se tiene certeza de que en algún momento morirá ${ }^{14}$. Lo anteriormente mencionado se refleja en los testimonios, pues los participantes sentían un alivio cuando las personas que estaban enfermas fallecían.

Estas experiencias construyen el concepto de la propia muerte en la persona mayor, ya que está mediada por los diversos factores mencionados antes, como el presenciar muertes cercanas, las enfermedades, el distanciamiento de los hijos y la familia, las separaciones, la jubilación, las pérdidas vividas y la elaboración de duelos correspondientes ${ }^{17}$. Asimismo, coincide con los resultados obtenidos, puesto que la pérdida de compañeros los prepara para su propia muerte.

Debido al impacto que genera la muerte de un residente, los diversos apoyos adquieren relevancia. Según Ghesquiere, las personas mayores confían en los soportes interpersonales existentes para ayudarse a manejar los síntomas de duelo ${ }^{16}$. Es en este momento cuando la persona mayor debe aprovechar todas las relaciones significativas que tenga, ya que son claves para lograr la recuperación emocional ${ }^{12}$.

De la misma forma, los diversos rituales de despedida se perciben como una oportunidad de expresar su sentir y de aliviar su propio dolor. Dentro de estos rituales destaca la importancia de las creencias religiosas, pues se considera que otorgan un espacio de despedida y, a su vez, permiten recordar con cariño y tranquilidad a quienes han partido. Según la literatura, las personas mayores enaltecen la importancia que la fe tiene en el afrontamiento del duelo, puesto que genera una instancia de regocijo ${ }^{18}$. Además, la creencia de que los fallecidos permanecen en un sitio donde descansan en paz también ayuda a aceptar la pérdida ${ }^{14}$.

Por lo expuesto anteriormente, el acompańamiento durante el proceso de muerte y duelo se traduce en que la persona mayor es capaz de reflexionar, con menos dolor y más objetividad, qué significa la pérdida y que dejó de aprendizaje, pues a partir de ella la propia vida se puede reestructurar y fortalecer ${ }^{14}$. Debido a ello, es necesario generar evidencia sobre esta problemática escasamente estudiada, pues el foco se encuentra en el duelo relacionado con el fallecimiento de la pareja.

De esta manera, se hace relevante y necesario para los profesionales de enfermería el poder comprender de manera integral la vivencia del duelo en personas mayores institucionalizadas cuando sufren la pérdida de un compañero residente. Además, se debe valorar y fortalecer el rol colaborador de las cuidadoras, las cuales tienen una función crucial en el momento de entregar apoyo. Más aún, es necesario fortalecer el trabajo en equipo con el propósito de brindar un ambiente y espacio propicio para vivenciar de manera saludable dicho proceso y, así, disminuir la recurrencia de duelos patológicos.

Este estudio permitió aportar a la comprensión de lo vivido por personas mayores institucionalizadas y, además, confirma la literatura y constituye un saber comprensivo que puede servir de guía para realizar cuidados de enfermería basados en las necesidades de cada residente ante el fallecimiento de sus compañeros. Además, se propone continuar investigando esta temática, siendo vinculada a la teoría de la subcultura postulada por Arnold Rose en $1965^{19}$. Por otra parte, es necesario mencionar que este estudio abarca población vulnerable tanto en el ámbito social como económico, lo que no se replica en la realidad de todos los hogares de acogida

\section{Conflicto de intereses}

Las autoras no presentan conflictos de intereses.

\section{- BIBLIOGRAFí́}

1. Cheng J, Lo R, Woo J. Anticipatory grief therapy for older persons nearing the end of life. Aging Health [Internet]. 2013 [citado 25 de febrero de 2019];9(1):103-14. http://dx.doi.org/10.2217/ahe.12.74

2. Hernández M, Oñate D, Rodríguez D, Sánchez L, Bezanilla J, Cam- pos J. El adulto mayor ante la muerte: análisis del discurso en el Estado de México. GEPU 2011:2(1):64-78.

3. Uribe A, Valderrama L. Actitud y miedo ante la muerte en adultos mayores. Pensamiento Psicológico. 2007;3(8):109-20.
4. Ministerio de Salud de Chile. Aprueba reglamento de establecimientos de larga estadía para adultos mayores. [Internet]. 2011 [citado 4 de junio de 2019]. Disponible en: https://www.leychile.cl/ Navegar?idNorma=1015936\&idVersion=2011-10-04 
5. Vergara S. Residencias para adultos mayores: criterios básicos para su adecuada selección. Revista Hospital Clínico Universidad de Chile [Internet]. 2008 [citado 20 de febrero de 2019]; 19:356-60. Disponible en: https://www.redclinica.cl/Portals/0/Users/014/14/14/ residencias_adultos_mayores.pdf

6. Streubert $\mathrm{H}$, Carpenter D. Qualitative Research in Nursing. Advancing the Humanistic Imperative. [Internet]. $5{ }^{\text {a }}{ }^{\text {ed. Philadelphia }}$ Lippincott; 2011 [citado 5 de noviembre de 2018]. Disponible en https://oysconmelibrary01.files.wordpress.com/2016/09/qualitative-research-in-nursing-advancing-the-humanistic-imp.pdf

7. Subsecretaria de Salud Pública. Manual de aplicación del Examen de Medicina Preventiva del Adulto Mayor. Santiago. [Internet] Disponible en: https://www.minsal.cl/portal/url/item/ab1f81f43ef0c2a6e04001011e011907.pdf

8. Noreña A, Alcaraz N, Rojas J, Rebolledo D. Aplicabilidad de los criterios de rigor y éticos en la investigación cualitativa. Aquichán [Internet]. 2012 [citado 20 de febrero de 2019]:12(3):263-74.
Disponible en: http://www.scielo.org.co/scielo.php?pid=\$165759972012000300006\&script=sci abstract\&tIng=es

9. Castillo E, Vásquez M. El rigor metodológico en la investigación cualitativa. Colombia Médica. 2003;34(3),164-67.

10. The National Commision for the Protection of Human Subjects of Biomedical and Behavioral Research. The Belmont Report: Ethical Principles and Guidelines for the protection of Human Subjects in research [Internet]. 1978 [citado 10 de enero de 2019] Disponible en: https://www.hhs.gov/ohrp/regulations-and-policy/belmont-report/read-the-belmont-report/index.html

11. Lolas F, Quezada A. Pautas éticas de investigación en sujetos humanos: nuevas perspectivas. Programa Regional de Bioética OPS/ OMS. 2003.

12. Villanueva P. Cuando la pareja se va. Reforma. 2014;27:8.

13. De Vries B. Grief: Intimacys reflection. Generations [Internet]. 2011 [citado 20 de febrero de 2019);25(2):75-80. Disponible en: https://www questia.com/library/journal/1P3-76555279/grief-intimacy-s-reflection
14. Montalvo G. La pérdida duele, pero luego pasará. Reforma. 2011 Oct 30:19.

15. Harwood D. Grief in old age. Rev Clin Gerontol. 2005;15(3-4):281-7.

16. Ghesquiere A. Patterns and outcomes of bereavement support-seeking among older adults with complicated grief and bereavement-related depression. Ann Arbor: Columbia University; 2012

17. Rodríguez A, Uribe F, Orbegozo LV, López S. Actitud y miedo ante la muerte en adultos mayores. Pensamiento Psicológico. 2007:3(8):109-20

18. Martin J. Spousal grief in older adults: The lived experience of surviving spouses during the second year of bereavement. Ann Arbor: Medical College of Ohio; 2003.

19. Merchán E, Cifuentes R. Teorías Psicosociales del Envejecimiento. Asociación Cicerón; 2014; 1-13. Disponible en: http://asociacionciceron.org/wp-content/uploads/2014/03/00000117-teorias-psicosociales-del-envejecimiento.pd 